\begin{tabular}{|c|c|c|c|}
\hline Eiszeitalter \begin{tabular}{c} 
Gegenwart \\
\hline
\end{tabular} & $\mathbf{2 8}$ & $\begin{array}{c}189-194 \\
3 \mathrm{Abb} .\end{array}$ & Öhringen/Württ. 1978 \\
\hline
\end{tabular}

\title{
Pollenanalytische Untersuchungen zur Entstehungs- und Entwicklungsgeschichte des Bruchbergmoores/Oberharz
}

\author{
BRIGITTE URBAN *) \\ Holocene, peat-bog, palynology, history of vegetation, environment Oberharz
}

$\mathrm{K}$ u rzf a s s ung : Auf drei kleinen benachbarten Moorflächen des Bruchberg-Moores wurden ein durchlaufendes Pollenprofil hergestellt und weitere Untersuchungen nächst der Torfbasis durchgeführt. Anhand der Untergrundmorphologie und der Basisproben konnte die flächenhafte Moorausbreitung rekonstruiert werden.

Die ältesten Vermoorungen setzen demnach im Präboreal ein. Unterbrechungen im Moorwachstum wurden am Ende der Waldzeiten VI (älteres Atlantikum) und in VIII b (Subboreal) gefunden.

\section{[Pollenanalytical Investigations of the Development of the Bruchbergmoor/Oberharz]}

A bstract: A traversing diagram of the pollen-content has been drawn up from one of three small patches of moor in the Bruchberg-Moor and further palynological analyses have been carried out near the lowermost parts of the peat-formation. The areal extension of the moor was reconstructed by the investigation of the subsurface relief and the results of pollenanalyses.

The oldest moorifications in this area started in the Preborial. Interruptions in the moorgrowth have been found to occur in the periods VI (early Atlanticum) and VIII b (Subboreal).

\section{Einleitung}

Beug (1957) machte auf das hohe Alter mancher Mittelgebirgsmoore aufmerksam. Ebenso fand WiLlutzKi (1962) im Oberharz weitere alte Vermoorungen, ließ aber erkennen, daß es sich möglicherweise nur um kleine Flächen spät- oder frühpostglazialer Entstehung handelt und großflächige Vermoorungen jüngere Bildungen sind.

Nachdem Schulz (1974) die großflächigen Vermoorungen des Bruchbergs anhand pollenanalytischer Untersuchungen waldgeschichtlich jüngeren Abschnitten zugeordnet hatte, lag die Vermutung nahe, auf den Hangterrassen und auf tiefer gelegenen Niveaus des Bruchberghanges ältere Bildungen finden zu können. Hierbei kam es darauf an, neben der vertikalen Entwicklung des Moores auch anhand von Torfbasisproben die flächenhafte Entwicklung zu erfassen.

Die ausgewählten drei Untersuchungsgebiete liegen am Südosthang des Bruchbergs im Oberharz in den Distrikten 201 und 200 (TK 25, Nr. 4228 und 4229) in einer Höhe zwischen $885 \mathrm{~m}$ und $910 \mathrm{~m}$ ü. NN (Abb. 1). Die Lage des Hauptprofils und der Profilpunkte der Fläche I, die im folgenden zu besprechen sind, gehen ebenfalls aus Abb. 1 hervor.

*) Anschrift der Verfasserin: Dipl.-Biol. B. U r b a n, Geologisches Institut der Universität zu Köln, Zülpicher Straße 49, D 5000 Köln 1. 


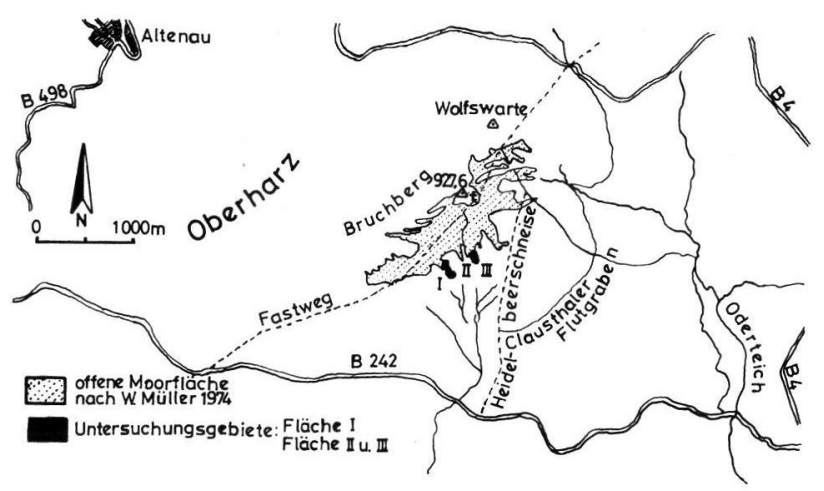

Abb. 1: Lage des Untersuchungsgebiets.

\section{Waldgeschichte}

Das in dem Profil I 18 lokalisierte durchlaufende Pollenprofil mit einer Mächtigkeit von $315 \mathrm{~cm}$ setzt im waldgeschichtlichen Abschnitt IV (Firbas 1949) ein ${ }^{1}$ ). Nach einem starken Birkenvorstoß beginnt an der Wende IV/V (Präboreal/Boreal) die nacheiszeitliche Wiederbewaldung mit dem Ausbreiten der Kiefer und Hasel. Im Laufe des Abschnittes ( $V$ a) nehmen die Anteile der Eichenmischwaldelemente zu, wobei die Ulme eine dominierende Stelle einnimmt. Die Überschneidung der EMW-Kurve mit der Pinus-Kurve fällt zusammen mit der rationellen Alnus-Grenze und dem weiteren Absinken des Anteils von Betula am Waldbild (Grenze V b/VI).

Der Abschnitt VI (älteres Atlantikum) ist durch die Dominanz des Eichenmischwaldes charakterisiert, wobei die Ulme Werte bis zu 28\% erreicht. Unter den Kräuterpollen ist Empetrum vorübergehend zusammen mit der Summe der übrigen Ericaceen stärker vertreten. Auch sind mit Beginn des Abschnittes VI erstmals Pollenkörner von Rhamnus frangula, Hedera belix und Viscum album gefunden worden. Ein vorübergehender Stillstand des Moorwachstums ist am Ende der Waldzeit VI zu fassen. Die organogene Ablagerung setzt im Abschnitt VIII a (Subboreal) wieder ein. Das erstmalige oder vermehrte Vorkommen von siedlungsanzeigenden Pflanzen und das Auftreten von Cerealia-Pollen im waldgeschichtlichen Abschnitt VIII a deuten auf Rodungs- bzw. Siedlungstätigkeit des prähistorischen Menschen in den Harzvorlandgebieten hin.

Während des Abschnittes VIII $b$ ist es ebenfalls nicht zur Torfbildung gekommen. An dieser Stelle befindet sich somit der zweite Hiatus im Pollenprofil. Erneutes Moorwachstum setzt in der Waldzeit IX a (älteres Subatlantikum) ein.

Die Zonen IX a und IX b werden durch die Vorherrschaft der Buche charakterisiert. Die Carpinus-Kurve steigt allmählich an (Maximum 12\%), jedoch bleibt im übrigen das Waldbild über diesen Zeitabschnitt hinweg unverändert. Unter den Nichtbaumpollen ist die Abnahme der siedlungsanzeigenden Pflanzen von Bedeutung, die durch die geschlossene Getreidekurve abgelöst wird (Grenze IX a/IX b).

In der nachfolgenden Siedlungszeit (X a und $\mathrm{X}$ b) wird der menschliche Eingriff in die natürliche Vegetationsentwicklung durch die frühgeschichtliche Besiedlung des Harzvorlandes deutlich.

Die stärkere Nutzung der Wälder zur Holzkohlegewinnung führt zu einer dadurch bedingten vermehrten Ausbreitung der Birke, und, was die Verheidung charakterisiert, auch zu einer starken Zunahme von Calluna (Firbas et al. 1939).

1) Aus technischen Gründen wurde von dessen bildlicher Wiedergabe abgesehen (vgl. URBAN 1975). 

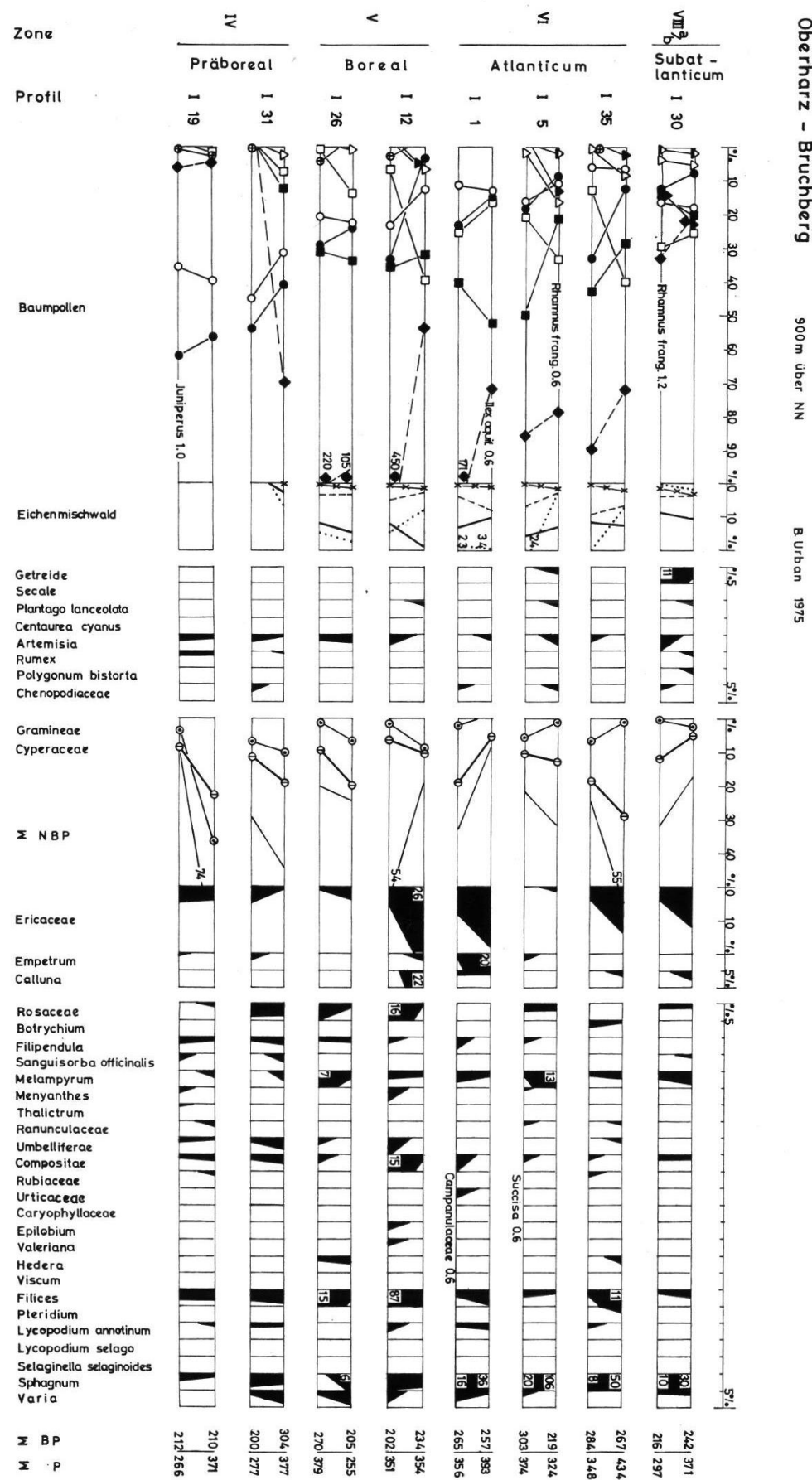

목
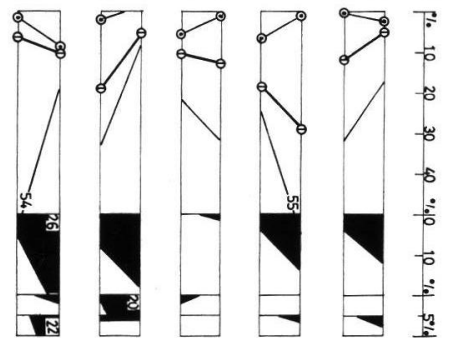

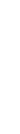


Da im Harz zur Verhüttung der Erze bevorzugt die Buche geschlagen wurde, kam es anthropogenbedingt zur Ausbreitung der Fichte $(\mathrm{X} \mathrm{b})$. Das Pollendiagramm bricht an der Grenze $\mathrm{X} \mathrm{a/X} \mathrm{b} \mathrm{ab,} \mathrm{wobei} \mathrm{anzunehmen} \mathrm{ist,} \mathrm{daß} \mathrm{jüngere} \mathrm{Ablagerungen} \mathrm{abgetragen} \mathrm{wurden,}$ da die heutige Oberfläche des Moores an der untersuchten Stelle stark zerstört und nicht von Pflanzen bewachsen ist.

Das kleine Moor (Fläche I, Abb. 3) hat an der Stelle des Hauptprofils seine größte Mächtigkeit und daher wurde hier sein Entstehungsgebiet vermutet. Seine Entwicklung, zumindest im Bereich des untersuchten Profilpunktes I 18, verlief über einen Schilftorf (Präboreal und Boreal) mit darauffolgendem Überwachsen durch einen Bruchwald (Boreal) weiter zum seggenreichen Niedermoor (Atlantikum) und schließlich zum Hochmoor hin (ab Ausgang Atlantikum).

Das Auftreten von zwei Hiati machte die Beschäftigung mit regionalen, bekannten Erscheinungen interessant. Für den markanten Hiatus unmittelbar vor dem Buchenanstieg (Grenze VIII a/VIII b) ist möglicherweise eine gute Korrelation gegeben. WILLUTZKI (1962) erwähnt unter anderem eine Rekurrenzfläche „Rfl. II“ (1500 C14-Jahre B.C.), die oberhalb der Grenze VIII a/VIII b, also zu Beginn des Fagus-Anstieges liegt.

Nimmt man ein klimatisch bedingtes Trockenfallen des Moores an - die einsetzende Verheidung, Zone VIII a) deutet auf eine Trockenphase hin - so liegt die Parallelisierung mit der „Rfl. II" der Vermoorung „Auf dem Acker“ (Willutzki 1962) nahe, da kleine abgeschlossene Moorkomplexe eher zur Austrocknung neigen als große zusammenhängende Moorflächen.

Die erste Unterbrechung steht am Beginn einer Zeit des Holozäns, die durch Instabilität sowohl hinsichtlich des Moorwachstums, der Bildung von Kalktuffen wie auch fluviatiler Bereiche gekennzeichnet ist (BRUNNACKER 1977).

Für den zweiten Hiatus findet sich keine regionale synchrone Erscheinung, doch die Stillstandsphase am Ende des Atlantikums (Zone VI) wird auf dieselbe Ursache zurückzuführen sein wie die soeben besprochene Entwicklung.

\section{Die horizontale Entwicklung der Moore}

Die in der Abb. 2 dargestellten Pollenspektren von Basisproben sind als Beispiele ausgewählt worden; es wurden bei der Datierung sämtlicher Basisproben zwischen zwei und vier Spektren (pro Probe) ausgezählt, die durchschnittlich einen vertikalen Abstand von $10 \mathrm{~cm}$ aufwiesen.

Das Gelände fällt von Profilpunkt (PP) I 1 nach PP I 22 terrassenförmig ab (Abb. 3), dabei liegt der PP I 16 an der Spitze eines nach Südosten geneigten Hanges. Der über die offene Moorfläche von Westen nach Südosten verlaufende Profilabschnitt durch die Punkte I 37 - I 28 zeigt eine Reliefdepression mit dem Zentrum im PP I 18. Das im Präboreal einsetzende Moorwachstum (Abb. 2 u. 3) konnte an solchen Punkten nachgewiesen werden, die sich durch Hanglage oder geschützte Lage in der flachen Hangdelle auszeichnen (Abb. 3 unterer Rand). An diesen ungefähr $900 \mathrm{~m}^{2}$ großen, ältesten Moorabschnitt schließt sich ein bis zu $10 \mathrm{~m}$ breiter Gürtel an, der in dem darauffolgenden waldgeschichtlichen Abschnitt V hangaufwärts vermoorte.

Ebenfalls während der Waldzeit V begann das Moorwachstum in einer Mulde und an einem kleinen Hang des von Norden nach Süden verlaufenden Bergrückens (PP I 10 und I 12). Im Atlantikum, Zone VI, war bereits die heute offene Moorfläche von Moorvegetation bedeckt, ebenso wie der gesamte terrassenförmige Bergrücken bis auf seinen tiefsten Teil. 


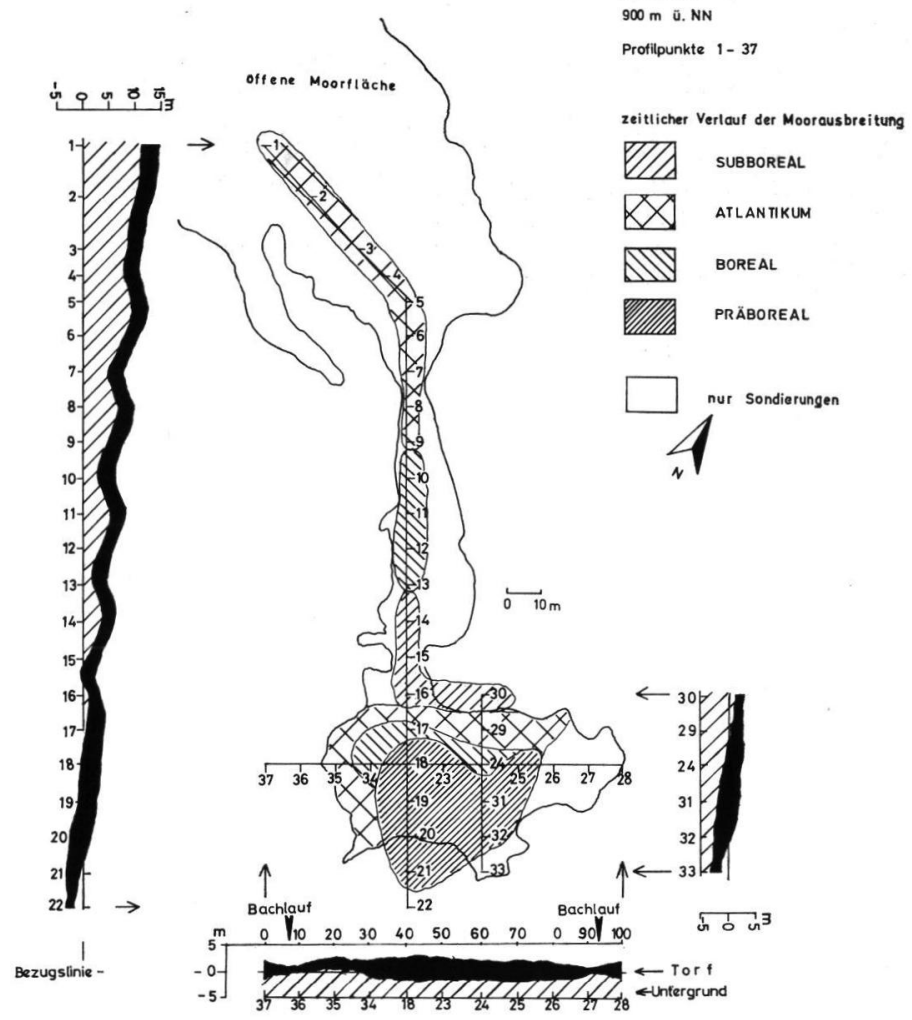

Abb. 3: Zeitlicher Ablauf der Moorausbreitung im Bereich der Fläche I. $\mathrm{Zu}$ den Profilen: Torfmächtigkeit $=$ schwarz, Untergrund $=$ schraffiert.

Es wurde bei der Diskussion des Hauptprofils schon auf den Ende des Atlantikum beginnenden und die Waldzeit VII umfassenden Hiatus hingewiesen. Auch bei der Analyse der Basisproben konnte beobachtet werden, daß es während dieser vegetationsgeschichtlichen Phase offenbar nicht zur horizontalen Moorausbreitung kam. Diese Entwicklung muß im Zusammenhang mit der Wasserführung gestanden haben. Die flächige Ausbreitung des kleinen Hangmoores ist anfänglich durch verstärktes Wachstum hangaufwärts, also dem Zug des Hangwassers entgegen, gekennzeichnet. Ursache ist die an dieser Front begünstigte Wasserversorgung der Moorvegetation.

Für die Moorflächen II und III, auf die hier nicht weiter eingegangen werden kann, trifft das besprochene Prinzip ebenfalls zu.

Durchgeführt wurde die Arbeit 1974/75 als Diplomarbeit im Botanischen Institut der Universität Köln in Zusammenarbeit mit dem Botanischen Institut der Universität Göttingen, Abteilung Palynologie, wo seither in diesem Bereich systematisch weitergearbeitet wurde. Herrn Professor U. Jensen möchte ich ebenso wie Herrn Professor Dr. H.-J. B e ug für die Betreuung und Unterstützung bei der Durchführung der Untersuchungen danken. 


\section{Schriftenverzeichnis}

BEUG, H.-J. (1957): Untersuchungen zur spätglazialen und frühpostglazialen Floren- und Vegetationsgeschichte einiger Mittelgebirge (Fichtelgebirge, Harz und Rhön). - Flora, 145: 167211; Jena.

BrunnaCKer, K. (1977): Das Holozän im Binnenland - die geologische Gegenwart. - Geol. Rdsch., 66: 755-770, 6 Abb., 1 Tab.; Stuttgart.

Firbas, F. (1949, 1952): Waldgeschichte Mitteleuropas. - Band I: 480 S.; Band II: 256 S.; Jena.

Firbas, F., Losert, H. \& Broihan, F. (1939): Untersuchungen zur jüngeren Vegetationsgeschichte im Oberharz. - Planta, 30: 256-422; Berlin.

Schulz, H. (1974): Untersuchungen über den Bruchberg/Oberharz im Hinblick auf die nacheiszeitliche Geschichte seiner Wälder und die Entwicklung seines Kamm-Hochmoores. - Unveröffentl. Staatsexamensarbeit; Göttingen.

Urban, B. (1975): Pollenanalytische Untersuchungen zur Entstehungsgeschichte des Bruchbergmoores/Oberharz. - Unveröffentl. Diplomarbeit; Köln.

WiLlutzKI, H. (1962): Zur Waldgeschichte und Vermoorung sowie über Rekurrenzflächen im Oberharz. - Nova Acta Leopoldina N. F., 25: Nr. 160; Leipzig.

Manuskript eingeg. 24. 11. 1977. 